\title{
Preparation, properties and applications of metal glass
}

\author{
Yunhe Yan and Shuqiong $\mathrm{Xu}^{\mathrm{a}}$ \\ School of Mechanical and vehicle Engineering, Linyi University, Shan Dong, China
}

\begin{abstract}
This paper reviews the definition and excellent properties of metal glass briefly. And it lists the preparation methods of metal glass. Furthermore, the urgent problems and the main research directions of metal glass are summarized, the development history of metal glass is reviewed, and the application direction of metal glass is sorted.
\end{abstract}

\section{Introduction}

Metal glass is a new material found in 1960s. Although metal glass is a member of the glass family, it is very different from the traditional glass material. Metallic glass is a kind of metastable metal material which is mainly composed of metal material(the atomic percentage of metal elements is $70 \%$ 80\%) and its atomic pile structure is long-range disorder, and it is made by modern rapid solidification metallurgy technology[1].

Metallic glass has some special properties of glass, metal, liquid and solid[2], so it has excellent performance and great application potential. For example, if placing the metal glass in a strong acid or strong base, it remains in perfect condition; The hardness of metal glass is similar to it of ceramics, but it can be as soft as plasticine at a certain temperature, flowing like liquid; A metal glass with diameter of $4 \mathrm{~mm}$ can suspend a weight of 3 tons. And metal glass is the most powerful armor material so far, which is one of the most ideal nano-processing materials[2]. What are the special properties and specific application direction of metal glass? How is it made? What is the main research on it? What is the history of metal glass research? This paper will focus on these problems and briefly introduce the metal glass.

\section{Performance and application of metal glass}

\subsection{Mechanical property}

The mechanical properties of metal glass are excellent, with high strength, high hardness, excellent elasticity and high wear resistance. In the military, the anti-tank kinetic energy piercing warhead, made of large metal glass and tungsten composite, has high strength and modulus, and has the self-sharpening effect[3]; In industrial production, high precision gear and bearing are available according to the high hardness of metal glass.
The superior wear resistance of metallic glass is shown in Figure 1, which makes metallic glass a surface coating material for various industrial equipment; In our life, massive metal glass shows low elastic modulus and large elastic limit, which is suitable for making some sports equipment, such as golf head, baseball bat, tennis racket, etc. On this basis, the commercial product of the zirconium foundation glass has been recognized by the market[4].

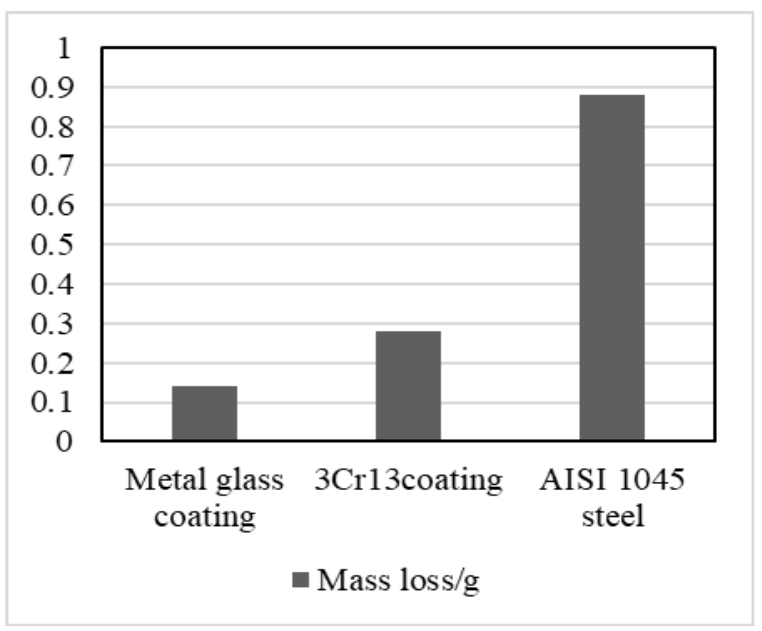

Figure 1. Comparison of abrasive resistance among amorphous FeCrBSiMnNbY coating, 3Cr13 coating and AISI 1045 Steel.[5]

In addition, metal glass also can be made into strips or lamination in the process, making tires, conveyors, cement products and reinforcing fiber for high pressure pipes. It can also be used to make various blades and security blades. Using amorphous alloy fibers instead of boron fibers and carbon fiber composite materials can further improve the adaptability of composites.

\subsection{Corrosion resistance}

Metal glass has strong corrosion resistance. For example, stainless steel in the solution containing chlorine ion is

\footnotetext{
a Corresponding author: xushuqiong11@sina.com
} 
generally going to pitting corrosion, intergranular corrosion and stress corrosion and hydrogen embrittlement, the amorphous $\mathrm{Fe} \mathrm{Cr}$ alloy-stainless steel can make up for these deficiencies[6], this makes the iron-based amorphous alloys is widely used in the oil, coal, steel and shipbuilding and other fields[7].

\subsection{Electrical property}

Metal glass has special electrical properties. Compared with crystalline alloy, the resistivity of metal glass is obviously improved, and the resistance temperature coefficient of metal glass is smaller than that of crystalline alloy. The low resistance temperature coefficient is helpful to eliminate thermal drift and integrate with temperature sensor. Therefore, metal glass becomes the best choice for developing multi-functional electronic skin, which can give intelligent robot more accurate "perception"[8].

\subsection{Soft magnetic properties}

Metallic glass can form a series of soft magnetic materials with excellent properties, among which the soft magnetism of the ferrous amorphous alloy material is the most attractive[9].The iron-based amorphous alloy is an excellent soft magnetic material, which is easily magnetized and demagnetized. It has better properties than normal crystalline magnetic material, which is shown in Table 1. Iron-based amorphous alloy is mainly used as the core material of transformer and motor. Because of the low magnetic loss, it can save a lot of power by replacing the silicon steel sheet with amorphous magnetic material.

Table 1. Comparison of properties between iron-based amorphous alloy and silicon steel.[10]

\begin{tabular}{|c|c|c|}
\hline Performance & $\begin{array}{c}\text { Iron-based } \\
\text { amorphous alloy }\end{array}$ & Silicon steel \\
\hline $\begin{array}{c}\text { Saturation } \\
\text { magnetic } \\
\text { induction }\end{array}$ & $1.54 \mathrm{~T}$ & $2.03 \mathrm{~T}$ \\
\hline Coercivity & $4 \mathrm{~A} / \mathrm{m}$ & $30 \mathrm{~A} / \mathrm{m}$ \\
\hline Resistivity & $130 \Omega \mu \mathrm{cm}$ & $0.45 \Omega \mu \mathrm{cm}$ \\
\hline $\begin{array}{c}\text { Maximum } \\
\text { permeability }\end{array}$ & $450000 \mathrm{Gs} / \mathrm{Os}$ & $40000 \mathrm{Gs} / \mathrm{Os}$ \\
\hline $\begin{array}{c}\text { Crystallization } \\
\text { temperature }\end{array}$ & $550^{\circ} \mathrm{C}$ & - \\
\hline $\begin{array}{c}\text { Curie } \\
\text { temperature }\end{array}$ & $415^{\circ} \mathrm{C}$ & $746^{\circ} \mathrm{C}$ \\
\hline Thickness & $30 \mu \mathrm{m}$ & $300 \mu \mathrm{m}$ \\
\hline
\end{tabular}

\subsection{Catalytic performance}

Metal glass has good catalytic performance. Amorphous alloys exhibit excellent catalytic performance in many reactions, especially in the catalytic hydrogenation of unsaturated compounds.

Metal glass can be used as a catalyst, such as amorphous cu-zr alloy catalyst, which has good catalytic hydrogenation for $\mathrm{CO}$ and $\mathrm{CO}_{2}$, the selectivity of hydrogenated methanol was higher than $90 \%$. In addition, the research indicates that both the ductile iron base and the magnesium base metal glass are also shown to have an incomparable advantage in the degradation of organic matter in sewage[11].

\subsection{Hydrogen storage property}

Some metal glass also has good hydrogen storage properties, which can be used as hydrogen storage material for vehicles with hydrogen as fuel. Use of hydrogen-fueled cars can reduce air pollution. The research of hydrogen storage materials stems from human needs for their own sustainable development. Hydrogen energy is a new energy source that is both efficient and clean, and the storage of hydrogen energy is the main bottleneck restricting the arrival of hydrogen energy society. The hydrogen storage capacity of amorphous alloys has attracted widespread attention. Zander et al.'s research shows that the hydrogen storage capacity of zr-based amorphous materials can be close to that of the best crystalline hydrogen storage materials[12]. However, amorphous alloy hydrogen storage materials are more concerned than crystalline hydrogen storage materials. The reasons include:

- The stress and strain produced by amorphous alloy in the process of hydrogen adsorption is relatively small, so the stability of hydrogen adsorption is better than that of crystalline alloy.

- There are many "defects" in amorphous alloys that resemble crystalline materials, which may provide more hydrogen sites and have higher hydrogen storage capacity.

- Amorphous atoms have a variety of glassy transitions that can provide more potential for hydrogen occupancy.

- Some alloy systems have a wide range of glass formation, which is conducive to the design of the chemical composition of the material, so the hydrogen storage performance has greater controllability.

\section{Preparation of metal glass}

In general, the metal material crystallizes during the cooling process, thus forming the steel and other crystalline materials that are common in our life. However, the rapid solidification can make the atoms of the molten metal have no time to form an ordered crystal structure in the solidification process, and prevent the formation of crystal phases in the solidification process. The disordered arrangement of the metal melt atoms is frozen[2], which forms metal glass. Therefore, metallic glass is also called "frozen melt". The fast cooling rate is the key condition for the preparation of metal glass. In theory, when the cooling rate is greater than the critical 
cooling rate of metal materials, any metal can be prepared into metal glass[13].

Because the critical cooling rate of the alloy materials of different components is different, the critical cooling rate of the metal glass directly influences the size of the material that can be prepared. The smaller the critical cooling rate, the easier it is to produce large size materials. Therefore, on the one hand, the researcher tries to improve the cooling speed of the equipment, on the other hand, they are also looking for a new alloy system to reduce the critical cooling rate. Here are some traditional method of preparing metal glass[6].

\subsection{Gas phase deposition}

The gas phase deposition method mainly includes sputtering and distillation. The metal glass produced by these two methods is only a small film and cannot be manufactured in industry. However, there are many kinds of metal glass which can be prepared by this method, so it only can be used for research.

\subsection{Liquid quenching}

The most commonly used liquid quenching method is the roller quenching method, which is divided into single roll and double roll. The process of single roll method is to put the test piece into the quartz tube, and the test block is melted by high frequency induction heating under the protection of argon gas. Then, let the argon blow the molten metal from the flat mouth at the bottom of the pass, and fall on the high speed rotating copper roller, forming a very thin amorphous band immediately after the rapid cooling. This method has been industrialized, and the non-alloy thin belt prepared by this method is used for transformer core.

\subsection{Chemical reaction method and solid reaction method}

In addition to liquid quenching and gaseous quenching, the main methods of preparing metallic glass include chemical reaction and solid reaction[14]. The solid reaction method has the advantages of simple equipment, easy industrialization and relatively wide range of alloy components, and the powder is easy to be formed. Solid reaction method can make the solid powder directly into the amorphous phase, for some by single roller quenching method cannot reach of amorphous alloys (e.g., A180Fe20), also realized the amorphous in ductile after 108h, thus expanding the scope of amorphous alloy composition. The disadvantage of the method is that the production efficiency is low and the alloying time is too long.

\subsection{Composite Process for Preparation of Amorphous Alloys}

The composite process is a development direction of the current RS-PM technology, which is to produce alloy powders through the dual action of rapid solidification and mechanical alloying. Mechanical alloying has at least two important functions for rapid solidification of powders. On the one hand, the mechanical properties of the material can be improved. On the other hand, the microstructure is more stable[15].

The composite process absorbs the strengths of both rapid solidification and mechanical alloying, complementing each other, thus promising to shorten the alloying time of the amorphous material, increase the amorphous transition temperature $T s$, increase the stability of the material's amorphous phase. There is no doubt that the use of composite processes to prepare amorphous alloys will have broad prospects.

\section{The main research problem and research direction of metal glass}

(1) In the future, we need to explore a new system with special properties and a new system with special features[15];

(2) Research on the preparation technology of block metal glass: the development process of metal glass is actually the development process of the preparation technology, and the breakthrough in this field is brought by the new preparation technology. The latest studies have shown that the lowest cooling speed, the largest diameter and the maximum reduced temperature are $0.001 \mathrm{~K} / \mathrm{s}, 100 \mathrm{~mm}$ and 0.75 respectively. Thus, the preparation of bulk metal glass has been largely free from the constraints of cooling speed and size[16];

(3) Brittle fracture and its mechanism and toughening of metal glass. Because there is no dislocation in the metallic glass, the deformation mechanism of the metallic glass is not yet clear, and there is no obvious tensile plasticity, which directly affects its application. Development of tough metal glass is a hot research topic;

(4) Structural characteristics and characterization methods: how to characterize the structural characteristics of metal glass is the key to understand and analyze the relationship between its composition, structure and performance;

(5) Glass forming ability of judgement and composition design: the exploring metallic glass materials is lack of effective and workable characterization of glass forming ability of judgment and composition design method. From the atomic level, structure, thermodynamics and dynamics quantifiable judgment, to set up the alloy design method is one of the basic research contents of metallic glass research field;

(6) The rheological properties of block metal glass: this is related to the superplastic deformation of metal glass and the important technology and application of its precision machining;

(7) Key scientific and technical problems in the practical application of metallic glass;

(8) Research on the nature of metal glass and glass transition, there is no unified and complete theory of glass transformation at present;

(9) Physical basis of excellent performance of 
block metal glass.

\section{Research history of metallic glass}

The earliest report on the study of metal glass in the history of science was that in 1934, Kramer discovered the amorphous metal film attached to the glass cold basement using evaporation deposition method[17]. Shortly thereafter, Brenner et al. prepared the Ni-p amorphous alloy by electrodeposition. In 1960, Clemen et al. created the first piece of only 20 um thick Au75Si25 metal glass chips by the melt quenching method and by X-ray diffraction method has proved its disordered atomic structure, created the history of metallic glass which was prepared by melt quenching[18]. However, the metal glass prepared by Clemen et al. is limited to strip, film and powder sample, and its smaller size limits the wide application of metal glass.

In the 1970s, the study of metal glass entered the active period. In 1971, Chen he-shou and other people adopted the fast cold continuous casting roll to form a series of thin strips and fillets of various iron based amorphous alloys, officially named "metallic glass". After 1980, Inoue et al. made a series of large metal glass by using the method of water cooling copper mold casting. Since then, a series of block metallic glasses have been developed, which greatly promoted the development of block metal glass[19]. A few years later, Peker and Lin developed bulk metallic glass, casting method to produce 50-100 - mm metal glass test bar and large size pieces, herald a larger size of metallic glass formation is promising. Subsequently, Allied Chemical Corporation of the United States developed a high-speed metal glass continuous production line at $2000 \mathrm{~m}$ per minute, for the first time in real sense to the purpose of commercializing metallic glass.

Into the 21st century, China National Iron and Steel Research Institute has also successfully developed a 10,000-ton amorphous strip production line. Some domestic enterprises and institutions are making key researches on the further commercial application of metallic glass.

\section{Conclusion}

Since 1960, metal glass has been developing for more than fifty years. Now, metal glass development prospects are very optimistic, and the fields involved are becoming more and more extensive. The metal-glass system is emerging in the field of chemical synthesis and fuel cell catalysis, and the research on the chemical functional application of metallic glass has just emerged. In addition, there is a great deal of exploration and research on nanocrystallization of metal glass, which is expected to make nanocrystalline of metallic glass into a new method for preparing nanomaterials[20]. However, it is a pity that people still do not know enough about the forming ability of large-sized metal glass, and the structure, performance and application of metal glass need to be further studied. In addition, the structure characterization, glass transition and deformation mechanism of amorphous are still unsolved mysteries in basic research.

\section{References}

1. Wang JunQiang, Functional application of metallic glass and related basic research [J]. Progress of Chinese materials,2014,(05):1674-3962.

2. Wang WeiHua. A brief history of metallic glass research [J]. physical,2011,(11):100190.

3. Xiao Jun. Development of super metal glass new material - new world of building materials industry [J]. Modern technology ceramics,2014,(02):442001.

4. Li YanZhuo. Mystery of unordered materials progress of metal glass research [J]. The journal nature, 2013,(03):100190.

5. Luo DaWei, Li CongBo, Chen WeiWei. Recent progress for metallic glass coatings[J]. Material guide,2015,(03):100081.

6. Qi Min, Yu yongsi. Mechanical engineering materials (10th edition) [M]. Dalian: DaLian university of technology press,2017.

7. Zhao GuoQiang, Zhang Song. Wear resistance and corrosion resistance of iron based amorphous coating $[\mathrm{J}]$. Rare metal materials and engineering,2016,(04):250061.

8. Xian HaiJie, Wang WeiHua. Amorphous alloy skin: new flexible strain sensor [J]. physical,2018,(01)

9. Han Ye, Zhu ShengLi, Inoue. Research progress of ferro-based soft magnetic materials amorphous alloy and block metal glass [J]. Functional materials,2016,(03):1001-9731.

10. Wang XiaoJun. The current situation of amorphous ally application[J]. Material guide,2006,(10)

11. Jia MengLu, Zheng YiXiong. Preparation and application of amorphous alloy catalyst [J]. Petrochemical technology,2015,(09):361021.

12. Lin HuaiJun, Zhu YunFeng, Liu YaNa, Li LiQuan, Zhu min. Progress in the Interaction of Amorphous Alloys with Hydrogen[J]. Chinese Journal of Physics.2017,(17):176105.

13. Lu WenHao. Properties, preparation and application of metallic glass [J]. The world of building materials,2017,(05):1674-6066.

14. Li ChuanFu, Zhang ChuanJiang, Xin XueXiang. Preparation and application of amorphous alloys [J]. Journal of ShanDong institute of light industry,2008,(01):1044-4280.

15. Wang WeiHua. Metallic glass [N]. Guangming Daily,2017,(04)

16. Fu MingXi, Li Yan, Zha YanQing. Research and application of large metal glass [J]. Material review,2005,(07):212013.

17. Yin Jun, Lei FuJun, Tan HeMiao. Classification of block metal glass [J]. Hot working process,2016,(14):1001-3814. 
18. Zhang HaiFeng, Ding BingZhe, Hu ZhuangQi. Research and progress of block metal glass [J]. Journal of metal,2001,(11):0412-1961.

19. Zhao LinZhi, Bai HaiYang. Study on rheological and relaxation behavior of metallic glass [D]. University of Chinese academy of sciences, 2017.

20. Liu XiongJun. Research progress on nanocrystalline mechanism of metallic glass [J]. Progress of Chinese materials,2014,(05):1674-3962. 